l'Association sanitaire würtembergeoise. La mort de tous deux a produit dans les rangs du Comité un vide douloureux.

Le 29 novembre, l'ancien président du Comité central, M. de Holleben, président de Cour d'appel, célébrait le 50 anniversaire de son entrée au service; à cette occasion on lui a remis une adresse de félicitations, artistement ornée et signée par tous les membres du Comité central, dans laquelle les services émineuts rendus par M. de Holleben à la Croix-Rouge ont été signalés avec reconnaissance.

La liste des articles de pansement, appareils, etc., qui est recommandée aux Sociétés de secours volontaires, pour leur servir de guide dans leurs acquisitions et les commandes qu'elles font, a été nouvellement révisée d'après les données des expériences scientifiques les plus récentes; elle a été ensuite revue par le ministère de la guerre, puis recommandée à l'attention des sociétés d'hornmes et des sociétés de femmes, par une circulaire commune du Comité central et de la Société patriotique des dames.

On s'occupe très activement de l'instruction d'infirmiers, qui a été entreprise par l'association des secoureurs volontaires organisée par la direction du Rauhe Haus, à Hambourg. Le nombre des hòpitaux et des cliniques dans lesquels ces secoureurs sont formés s'est beaucoup accru; l'intérêt qu'inspire l'association va tous les jours croissant, et, notamment parmi les cercles d'étudiants, de nombreuses marques de sympathie lui ont été données. Si, d'après ses débuts, il est possible de tirer une conclusion pour l'avenir, on peut avoir le meilleur espoir du développement futur de l'œenvre de secours volontaire aux malades, car un grand nombre d'hommes instruits, poussés par les motifs les plus désintéressés, sont gagnés à cette œuvre.

\title{
BAVIERE
}

\section{PROTECTORAT DE LA SOCIÉTÉ BAVAROISE}

Le Comité central bavarois nous communique la lettre suivante, adressée à son premier président, par laquelle S. A. R. le prince 
régent de Bavière, a daigné consentir à devenir le protecteur de la Société bavaroise de secours aux militaires blessés et malades.

- C'est avec un intérêt tout spécial que j'ai pris connaissance du rapport qui m'a été présenté par le Comité central de la Société de secours aux militaires blessés et malades, sur les travaux multiples de la Société depuis sa fondation, et c'est avec une joie profonde que j'ai pu constater que cette Société, qui, depuis vingt années, remplit la noble tâche qu'elle s'est proposée, continue à obtenir des résultats riches en bénédictions. Aussi, est-ce avec plaisir que j'accède à la demande "qui m'a été faite par le Comité central d'accepter le protectorat de la Société, et je saisis cette occasion pour exprimer, à tous ses organes et à ses membres, ma reconnaissance pour l'activité dévouée et infatigable qu'ils ont déployée jusqu'à présent. '

Berchtesgaden, 28 octobre 1886.

LUITPOLD,

Prince de Bavière.

\section{BELGIQUE}

UNE PAGE DE L'Histoire DE LA GONVENTION DE GENÈVE, PAR G. MOYNIER

Désireux de ne laisser ignorer à nos lecteurs aucune des publications qui touchent à la Croix-Rouge, nous leur signalerons aujourd'hui un article qui vient de paraître dans la Revue de droit international et de législation comparée '. Son auteur, M. Gustave Moynier, y a retracé les actes les plus récents relatifs à la Convention de Genève, c'est-à-dire d'une part l'adhésion du Japon, et d'autre part les expériences de la guerre serbo-bulgare. En y jetant les yeux, les lecteurs de notre Bulletin se convaincront sans peine que ce n'est pas pour eux que cet exposé a été écrit, car il ne contient aucune donnée historique qu'ils n'aient pu lire antérieu-

${ }^{1}$ Bruxelles et Leipzig, Librairie Muquardt, 1886, $n^{\circ} 6$. 